\title{
Which is better to preserve pulmonary function: Short-term or prolonged leukocyte depletion during cardiopulmonary bypass?
}

\author{
Kaiyu Tao, MD, ${ }^{\text {a,* }}$ Qi An, MD, ${ }^{\text {a, } *}$ Ke Lin, MD, ${ }^{\text {a }}$ Raphael C. Lui, MD, FACS, ${ }^{a}$ Xiaodong Wu, MS, ${ }^{b}$ \\ Jing Zhou, MS, ${ }^{c}$ and Lei Du, MD ${ }^{\mathrm{b}}$
}

\begin{abstract}
Objectives: Neutrophils are crucial in the development of acute lung injuries during cardiopulmonary bypass. However, the efficacy of leukocyte depletion on pulmonary protection remains controversial, possibly owing to different filtration strategies used in the literature. In this study, we investigated whether short-term leukocyte depletion strategy is more efficacious than prolonged leukocyte depletion in preserving pulmonary function.
\end{abstract}

\begin{abstract}
Methods: Eighteen adult dogs were randomized equally into 3 groups. Leukocyte-depleting filters were used for 10 minutes in the LD-S group, throughout cardiopulmonary bypass in the LD-T group, and not used in the control group. Neutrophil counts, elastase, and interleukin- 8 concentrations in plasma, myeloperoxidase and interleukin8 concentrations in pulmonary tissue, and pulmonary vascular resistance and oxygen index were determined to evaluate the inflammatory response and damage to pulmonary function.
\end{abstract}

Results: Although the neutrophil count and pulmonary parenchymal myeloperoxidase contents were significantly lower in both LD-S and LD-T groups than that in the control group, lower pulmonary parenchymal interleukin-8 level, lower pulmonary vascular resistance $\left(113 \pm 33\right.$ dyne $\left.\cdot \mathrm{s} / \mathrm{cm}^{5}\right)$, higher oxygen index $(366 \pm 82.3$ $\mathrm{mm} \mathrm{Hg}$ ), and thinner alveolus wall thickness were seen only in the LD-S group, and the pulmonary parenchymal interleukin-8 levels were also lower in the LD-S group after cardiopulmonary bypass. The plasma elastase and interleukin-8 levels were significantly lower in the LD-S group, but they were significantly higher in the LD-T group compared with the control group after cardiopulmonary bypass.

Conclusions: Short-term rather than prolonged leukocyte depletion during cardiopulmonary bypass appears to be more efficacious in protecting pulmonary function via attenuation of the extracorporeal circulation-induced inflammatory response. (J Thorac Cardiovasc Surg 2009;138:1385-91)

Acute lung injury (ALI) is common after cardiopulmonary bypass $(\mathrm{CPB})$, frequently causing prolonged intensive care unit and hospital stay. ${ }^{1}$ It is believed that neutrophil sequestration in the pulmonary microcirculation with subsequent activation and release of proinflammatory cytokines, proteases, and oxygen-derived free radicals play a crucial role in CPB-induced ALI. ${ }^{2}$ It is therefore rational to speculate that leukocyte depletion will be effective in attenuating CPB-induced ALI. However, depending on the strategy and extent of leukocyte depletion, the results were controversial. ${ }^{3-7}$ Although the depletion filter effectively removed the leukocytes from the circulation, it did not inhibit the activation of the leukocytes trapped in the filter membrane, ${ }^{8}$

\footnotetext{
From the Departments of Thoracic and Cardiovascular Surgery, ${ }^{\mathrm{a}}$ Anesthesiology, ${ }^{\mathrm{b}}$ and Laboratory Medicine, ${ }^{c}$ West China Hospital, Sichuan University, Chengdu, Sichuan, China.

Funded by the National Natural Science Foundation of China (NSFC, 30400439, 30672078); Key Project of Chinese Ministry of Education (106132).

* K.T and Q.A contributed equally to the study.

Received for publication April 28, 2009; revisions received June 29, 2009; accepted for publication July 23, 2009; available ahead of print Oct 15, 2009.

Address for reprints: Lei Du, MD, Department of Anesthesiology, West China Hospital, Sichuan University, Chengdu, Sichuan, 610041, People's Republic of China (E-mail: dulei1972@yahoo.cn).

$0022-5223 / \$ 36.00$

Copyright (c) 2009 by The American Association for Thoracic Surgery

doi:10.1016/j.jtcvs.2009.07.059
}

thus allowing them to continuously release elastase and other proinflammatory factors with subsequent magnification of the inflammatory process resulting in ALI. Therefore, leukocyte-depleting strategies, ${ }^{6,9}$ especially the duration of depletion, may be critical for attenuation of inflammation and organ protection. ${ }^{10}$ In the present study, we investigated whether short-term leukocyte depletion was more efficacious than prolonged depletion during CPB in attenuating systemic inflammatory response and thus preserving pulmonary function.

\section{MATERIALS AND METHOD}

\section{Animals, Surgical Technique, and CPB}

The study was approved by the hospital animal ethics committee and all animals in the study received humane care in compliance with the "Guide for the Care and Use of Laboratory Animals." Eighteen healthy adult mongrel male dogs, weighing from 17.5 to $25.0 \mathrm{~kg}$, were provided by The Animal Experiment Center of Sichuan University. Anesthesia was administered by intraperitoneal injection of sodium pentobarbital $(25 \mathrm{mg} / \mathrm{kg})$ and maintained with intravenous injection of midazolam $\left(0.05 \mu \mathrm{g} \cdot \mathrm{kg}^{-1} \cdot \mathrm{min}^{-1}\right)$, fentanyl citrate $\left(0.02 \mu \mathrm{g} \cdot \mathrm{kg}^{-1} \cdot \mathrm{min}^{-1}\right)$, and pipecuronium bromide $(0.02-0.04 \mathrm{mg}$ $\left.\cdot \mathrm{kg}^{-1} \cdot \mathrm{h}^{-1}\right)$. After intubation with a $7.5 \mathrm{~F}$ endotracheal tube, each dog was mechanically ventilated (Datex-Ohmeda Excel 210; Soma Technology, Cheshire, Conn) with a tidal volume of $15 \mathrm{~mL} / \mathrm{kg},{ }^{11}$ fraction of inspired oxygen of 0.5 , and a respiratory rate of 12 breaths $/ \mathrm{min}$. Arterial blood gases were determined by i-STAT portable clinical analyzer (i-STAT Corporation, Cranbury, NJ) every hour. Carbon dioxide tension was 


$$
\begin{aligned}
& \text { Abbreviations and Acronyms } \\
& \begin{aligned}
\text { ALI } & =\text { acute lung injury } \\
\text { CPB } & =\text { cardiopulmonary bypass } \\
\text { ELISA } & =\text { enzyme-linked immunosorbent assay } \\
\text { IL- } 8 & =\text { interleukin } 8 \\
\text { MPO } & =\text { myeloperoxidase } \\
\text { NE } & =\text { neutrophil elastase } \\
\text { PVR } & =\text { pulmonary vascular resistance }
\end{aligned}
\end{aligned}
$$

maintained between 30 and $40 \mathrm{~mm} \mathrm{Hg}$ throughout the experiment by adjusting the respiratory rate, and $\mathrm{pH}$ was maintained between 7.35 and 7.45 by intravenous injection of sodium bicarbonate. The left femoral vein and right femoral artery were catheterized for blood sample withdrawal and monitoring of arterial pressure (Philips M1026; Philips Electronics North America Corporation, Andover, Mass). A pulmonary artery flow-directed catheter was placed through the right external jugular vein for determination of cardiac output (Abbott Critical Care Systems, Chicago, Ill) by the thermal dilution method. An esophageal temperature probe was inserted to measure core temperature. The electrocardiogram was continuously monitored throughout the experiment (Philips M1026).

The heart was exposed by a median sternotomy. After systemic heparinization $(3 \mathrm{mg} / \mathrm{kg})$, an $18 \mathrm{~F}$ aortic cannula was inserted into the distal ascending aorta and a $30 \mathrm{~F}$ venous cannula into the right atrium to complete $\mathrm{CPB}$ circuitry. The CPB circuitry included a bubble oxygenator (95 type; Xi-Jing Medical Ltd, Xi'an, China), a regular arterial filter (pore size $40 \mu \mathrm{m}$; Xi-Jing Medical Ltd), a roller pump (Stöckert II, Munich, Germany), and connection tubings. The pump was primed with Ringer lactate solution $(750 \mathrm{~mL}), 6 \%$ sodium chloride (Voluven; $750 \mathrm{~mL}$ ), heparin $(20 \mathrm{mg}$ ), and $5 \%$ sodium bicarbonate $(50 \mathrm{~mL})$. The activated coagulation time was maintained over 480 seconds throughout perfusion. The flow rate of CPB was maintained at 60 to $80 \mathrm{~mL}$ $\cdot \mathrm{kg}^{-1} \cdot \mathrm{min}^{-1}$ and mean arterial pressure between 50 and $80 \mathrm{~mm} \mathrm{Hg}$. After 10 minutes of stable $\mathrm{CPB}$, the heart was arrested at a body temperature of $32{ }^{\circ} \mathrm{C}$ for 60 minutes by hyperkalemic cold blood cardioplegic solution $(30$ $\mathrm{mL} / \mathrm{kg}$, 4 parts blood/1 part crystalloid cardioplegic solution; potassium concentration: $20 \mathrm{mmol} / \mathrm{L}$ ), then reperfused for 30 minutes before weaning from CPB. Postoperatively, the dogs were observed for 24 hours under anesthesia and controlled ventilation before they were humanely killed with an intravenous bolus injection of sodium pentobarbital $(120 \mathrm{mg} / \mathrm{kg})$.

\section{Protocol}

The dogs were equally randomized into 3 groups according to a computer-generated random number $(n=6$ in each group). In the LD-T group, the leukocyte depletion filter (Heart-Care System; Separator Haemo-Technology Beijing Co Ltd, Beijing, China) was incorporated in the CPB circuitry between the arterial and venous lines throughout CPB. In the LD-S group, the filter was incorporated in the CPB circuitry for only 10 minutes after the initiation of CPB. There was no leukocyte filter in the control group. At the end of CPB, only the skin but not the sternum was closed to allow for the subsequent repeated lung biopsy specimens to be obtained. The surgeons, anesthesiologists, pathologists, and laboratory staff were blinded until the data were all collected.

\section{Neutrophil Counts and Plasma Neutrophil Elastase and Interleukin-8 Levels}

So that the efficacy of leukocyte depletion could be assessed, blood samples were collected from the femoral vein for analysis of the neutrophils by a hematology automated analyzer (Sysmex XE-2100; Sysmex Corporation, Nishi-ku, Japan) within 2 hours from the time of collection. Plasma concentration of interleukin-8 (IL-8) and neutrophil elastase (NE) were quantified.
Heparinized blood samples were collected from the femoral vein before, at the end of, and 3,6,12, and 24 hours after termination of CPB. The samples were immediately centrifuged with $1500 \mathrm{rpm}$ for 15 minutes at $4{ }^{\circ} \mathrm{C}$ (Biofuge Stratos, Heraeus, Germany), and the plasma was then removed to another tube and stored at $-80^{\circ} \mathrm{C}$. The IL- 8 levels were determined by two-site sandwich enzyme-linked immunosorbent assay (ELISA) developed by Bionewtrans Pharmaceutical Biotechnology Co, Ltd. (Franklin, Mass), according to the manufacturer's instruction.

\section{Leukocyte Sequestration and Inflammatory Factors in Lung Tissue}

Lung tissue portions $(0.5 \mathrm{mg}$ each) were harvested from the right upper, middle, and lower lobes and from the left upper and lower lobes of the lung before, at the end of, and 3, 6, 12, and 24 hours after the initiation of CPB. The samples were immediately stored in liquid nitrogen until ready to be analyzed. For analysis, the samples were homogenized and dissolved in $1 \mathrm{~mL}$ potassium phosphate. The tube with $1 \mathrm{~mL}$ potassium phosphate was weighed before and after dissolving the tissue. The supernatant was collected in phosphate buffer after centrifugation (1500 rpm for 15 minutes at $\left.4^{\circ} \mathrm{C}\right)$.

The concentrations of myeloperoxidase (MPO) and IL-8 in the supernatant were determined by ELISA, and the real concentration of these factors in the lung tissues was calculated with the following equation: $\mathrm{C}_{\text {real }}=\mathrm{C}_{\mathrm{ELISA}} \times 1 \mathrm{~mL} /$ tissue weight $\left(\mathrm{C}_{\text {real }}\right.$, real concentration in pulmonary tissue; $\mathrm{C}_{\mathrm{ELISA}}$, concentration in supernatant determined by ELISA; tissue weight, weight of tissue dissolved in $1 \mathrm{~mL}$ of potassium phosphate).

\section{Hemodynamics and Respiratory Function}

The hemodynamic data were collected and arterial blood gases were analyzed before, at the end of, and 3, 6, 12, and 24 hours after the termination of CPB. Pulmonary vascular resistance (PVR) was measured to estimate pulmonary vascular status by the Abbott Critical Care Systems. Respiratory function was evaluated by oxygen index by the following formula:

$$
\mathrm{OI}=\mathrm{PaO}_{2} / \mathrm{FiO}_{2}
$$

where $\mathrm{OI}=$ oxygen index, $\mathrm{PaO}_{2}=$ arterial oxygen pressure, and $\mathrm{FiO}_{2}=$ fraction of inspired oxygen.

\section{Pathologic Examination}

The lung tissue samples, about $0.5 \mathrm{mg}$ each, were harvested from the right upper, middle, and lower lobes, as well as from the left upper and lower lobes, within 24 hours after $\mathrm{CPB}$. The samples were stained within 24 hours with hematoxylin and eosin. The portion of the pulmonary parenchyma 100 $\mu \mathrm{m}$ away from the cut margin was examined under a light microscope. The thickest portion of every alveolar wall in five different visual fields was recorded for evaluation of the alveolar wall thickness in a slide.

\section{Statistical Analysis}

Blood cell counts, the cytokines, and postoperative observational data were expressed as means $\pm \mathrm{SD}$. The data were analyzed with 1-way analysis of variance with Student-Newman-Keuls test by the SPSS for Windows V15.1 statistical software (SPSS, Inc, Chicago, Ill).

\section{RESULTS}

\section{Both Leukocyte Removal Strategies Effectively Reduced Neutrophil Counts}

The neutrophil count was similar among the 3 groups before leukocyte depletion. After 10 minutes of leukocyte depletion on $\mathrm{CPB}$, the circulating neutrophils decreased significantly to $1.23 \pm 0.168 \times 10^{9} / \mathrm{L}$ in the 
LD-T group (baseline, $4.02 \pm 1.52 \times 10^{9} / \mathrm{L} ; P<.001$ ) and $1.05 \pm 0.225 \times 10^{9} / \mathrm{L}$ in the LD-S group (baseline, $4.9 \pm$ $\left.1.7 \times 10^{9} / \mathrm{L} ; P<.001\right)$, which were significantly lower counts than that in the control group $\left(4.06 \pm 2.4 \times 10^{9} / \mathrm{L} ; P=.003\right.$, Figure 1). The neutrophil count increased in all 3 groups after CPB. Compared with the control group, the neutrophil counts were significantly lower in the LD-T and LD-S groups before aortic unclamping (75 minutes during $\mathrm{CPB}$ ), at the end of CPB, and even 3 hours after CPB. However, the neutrophil counts were similar in the 2 leukocyte depletion groups during and after CPB $(P>.05)$.

\section{The Influence of Different Leukocyte Depletion Strategies on Pulmonary Function}

PVR was measured to estimate pulmonary vessel status. Figure 2, $A$, shows that the PVRs in the LD-S group (73 \pm 21.6, $215 \pm 69,204 \pm 44.7,124 \pm 38$, and $113 \pm 33$ dyn - $\mathrm{s} / \mathrm{cm}^{5}$, respectively) were significantly lower than those in the control group $(215 \pm 80.9,337 \pm 155,383 \pm 105$, $228 \pm 76.8$, and $223 \pm 26.7 \mathrm{dyn} \cdot \mathrm{s} / \mathrm{cm}^{5}$, respectively) and the LD-T group $(164 \pm 107.9,321 \pm 123.6,283 \pm$ $81.8,262 \pm 104.4$, and $314 \pm 151 \mathrm{dyn} \cdot \mathrm{s} / \mathrm{cm}^{5}$, respectively) at the end of and 3, 6, 12, and 24 hours after CPB. However, the PVR in the LD-T group (314 $\left.\pm 151 \mathrm{dyn} \cdot \mathrm{s} / \mathrm{cm}^{5}\right)$ was significantly higher than that in the control group (223 \pm $26.7 \mathrm{dyn} \cdot \mathrm{s} / \mathrm{cm}^{5}$ ) at 24 hours after CPB. In the present study, the oxygen index was determined to estimate the status of respiratory function. The oxygen indexes of 3 groups de- creased significantly, but no significant differences were noticed among the 3 groups throughout the study until 24 hours after CPB, when the oxygen index in the LD-S group (366 $\pm 82.3 \mathrm{~mm} \mathrm{Hg})$ was significantly higher than that in the LD-T group $(204 \pm 79.2 \mathrm{~mm} \mathrm{Hg})$ at 24 hours after CPB $(P=.021$, Figure 2, $B)$.

\section{The Impact of Different Leukocyte Depletion}

\section{Strategies on the Plasma Inflammatory Mediators}

Although the leukocyte filter significantly reduced circulating neutrophils, the concentration of NE, a marker of neutrophil activation, was significantly higher in the LD-T group than that in the control group after CPB $(P<.01)$. However, it was lower in the LD-S group than in the control group at 3 and 6 hours after $\mathrm{CPB}(P<.05$, Figure $3, A)$. Figure $3, B$, showed the plasma concentrations of IL- 8 in the 3 groups. Similarly, the IL- 8 levels in the LD-T group were significantly higher than those in the control group at the end of and 3 and 6 hours after CPB $(P<.05)$. It was also higher than that in the LD-S group at the end of and 3 and 6 hours after CPB $(P<.01)$. There was no difference between the IL- 8 concentrations of the LD-S group and the control group throughout the study.

\section{Only Short-Term Leukocyte Depletion Attenuates Pulmonary Inflammation}

The concentration of MPO in pulmonary tissue was determined for evaluating the sequestration of neutrophils

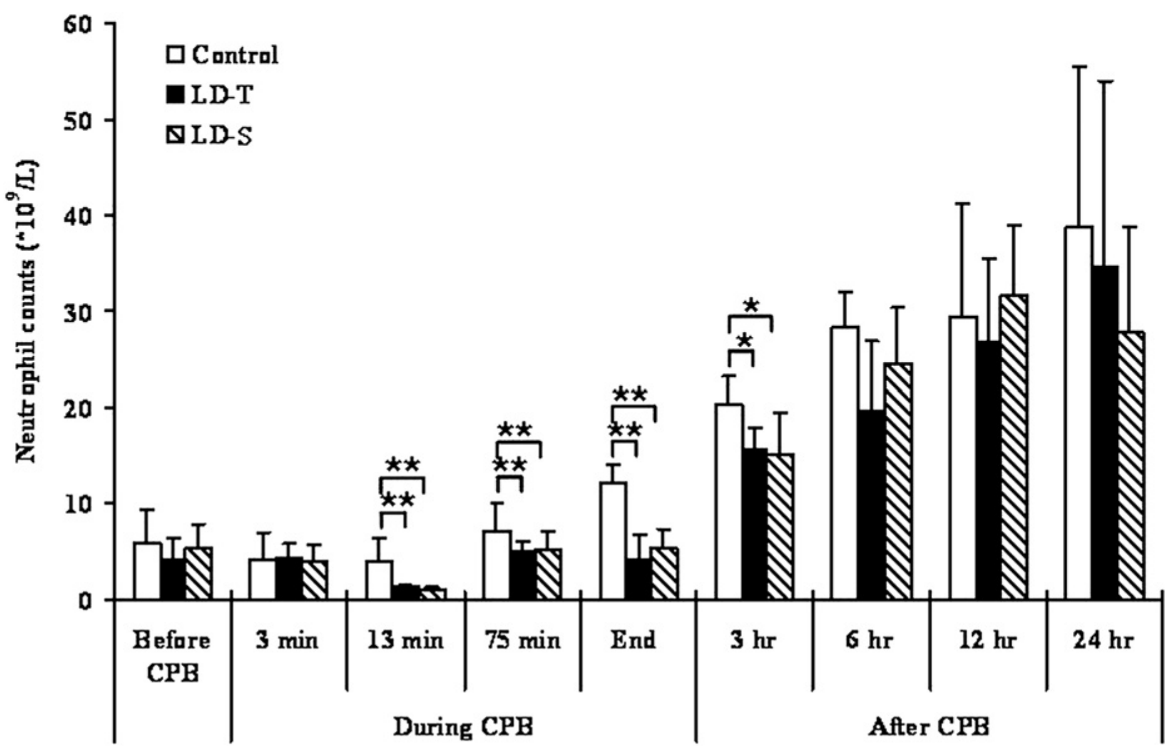

FIGURE 1. Mean neutrophil counts of the 3 groups. No difference of neutrophil counts was observed among the 3 groups before filtration ( 3 minutes of $\mathrm{CPB})$. The counts were significantly lower in the LD-T and LD-S group compared with the control group $(P=.003$ and $P=.002$, respectively) at 13 minutes of CPB. The neutrophil counts in the LD-T and LD-S groups were less than in the control group until 3 hours after CPB. There was no difference between the LD-T and LD-S groups. $L D-T$ group, The leukocyte depletion filter was used from 3 minutes to the end of CPB; $L D-S$ group, the leukocyte depletion filter was used from 3 minutes to 13 minutes of $\mathrm{CPB}$; control group, no leukocyte depletion filter was used; $C P B$, cardiopulmonary bypass. $* P<.05$; ** $P<.01$ versus control. 

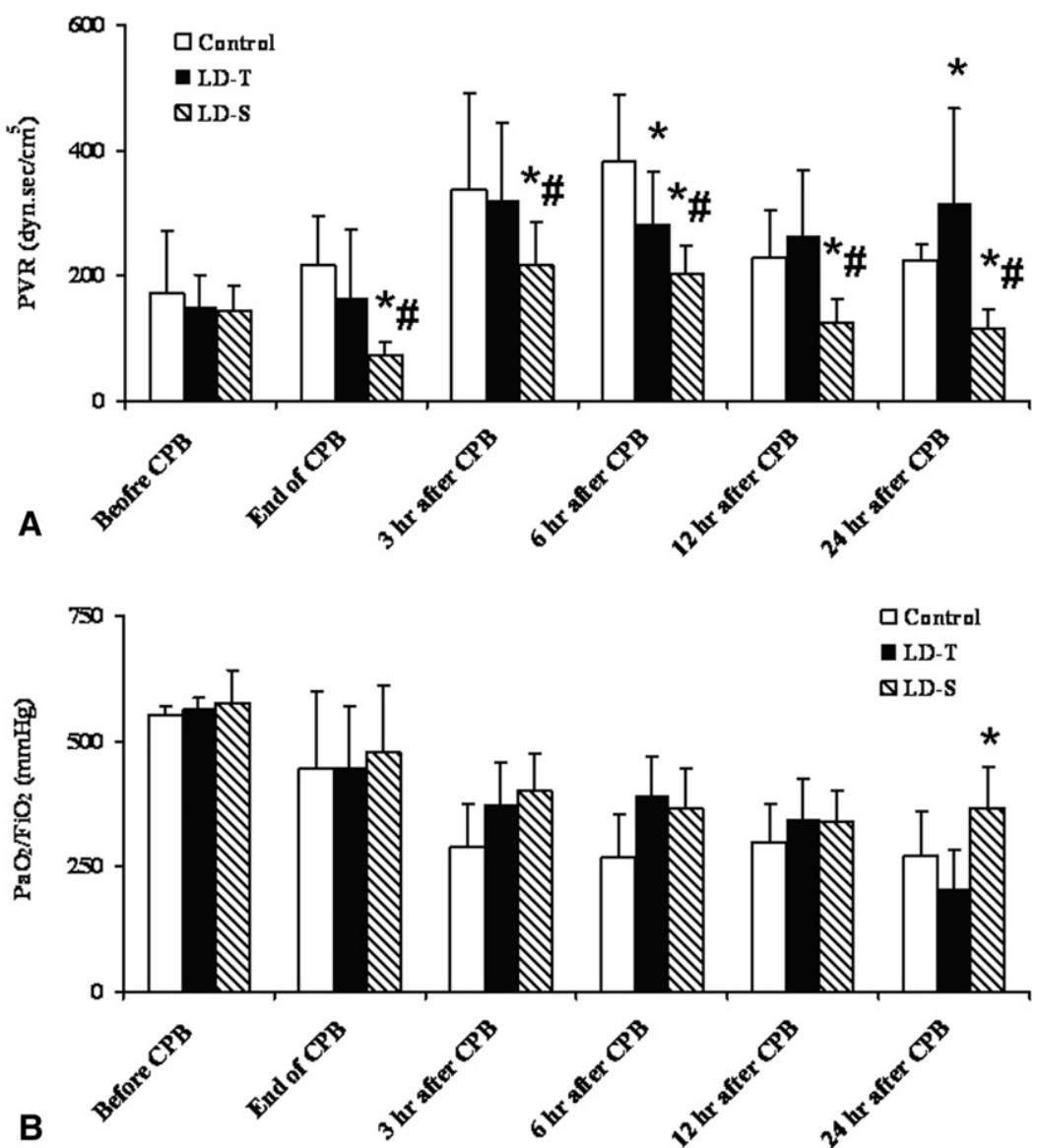

FIGURE 2. The respiratory status of the 3 groups. A, The mean PVR in the LD-S group was significantly lower than that in the control group and LD-T group at the end of and 3, 6, 12, and 24 hours after CPB $(P<.05$, respectively), but the PVR in the LD-T group at 24 hours after CPB $(P<.05)$ was significantly higher than that in the control group. B, Oxygen index in the LD-S group was significantly higher than that in the LD-T group at 24 hours after CPB $(P=.048) . L D-T$ group, The leukocyte depletion filter was used from 3 minutes to the end of CPB; $L D-S$ group, the leukocyte depletion filter was used from 3 minutes to 13 minutes of $\mathrm{CPB}$; control group, no leukocyte depletion filter was used; $C P B$, cardiopulmonary bypass; $P V R$, pulmonary vascular resistance; $\mathrm{PaO}_{2}$, arterial oxygen tension; $\mathrm{FiO}_{2}$, fraction of inspired oxygen. $* P<.05$ versus control group; $\# P<.01$ versus LD-T group.

in the lungs. Figure 3, $C$, shows that the concentrations of MPO in the LD-T and LD-S groups were lower than that in the control group from the end of CPB until 6 hours after $\mathrm{CPB}$. Interestingly, the concentration of MPO in the LD-T group increased at 24 hours after $\mathrm{CPB}$, and it was significantly higher than that in the control and LD-S groups. The pulmonary tissue concentration of IL-8 in the LD-S group was significantly lower than that in the LD-T group from the end of CPB until 24 hours after CPB $(P<.01)$, and it was also significantly lower than that in the control group from the end of CPB until 6 hours after CPB $(P<.01$, Figure $3, D)$.

\section{The Impact of Different Leukocyte Depletion Strategies on Alveolar Wall}

The pulmonary tissue was examined under a light microscope with a $400 \times$ magnification factor. The average alveolar wall thickness in the control group $(10.2 \pm 3.1$ $\mu \mathrm{m})$ and LD-T group $(9.7 \pm 3.0 \mu \mathrm{m})$ was significantly thicker than that in the LD-S group $(6.5 \pm 2.3 \mu \mathrm{m} ; P<$ $.001)$ at 24 hours after CPB, whereas no significant difference was noticed between the LD-T and control groups $(P=.56$, Figure 4$)$.

\section{DISCUSSION}

$\mathrm{CPB}$, although an essential tool for cardiac surgery, often causes ALI, even adult respiratory distress syndrome postoperatively, ${ }^{12}$ with mortalities that can be as high as $53 \%{ }^{13}$ It has been reported that leukocytes play an important role in the occurrence of ALI. ${ }^{14}$ Therefore, it is rational to speculate that leukocyte depletion during CPB should reduce the incidence and extent of ALI. However, the efficacy of leukocyte depletion to attenuate CPB-induced ALI is conflicting. ${ }^{3-7}$

In our study, the neutrophil removal rate for short-term depletion was $74.33 \%$ when compared with that before 

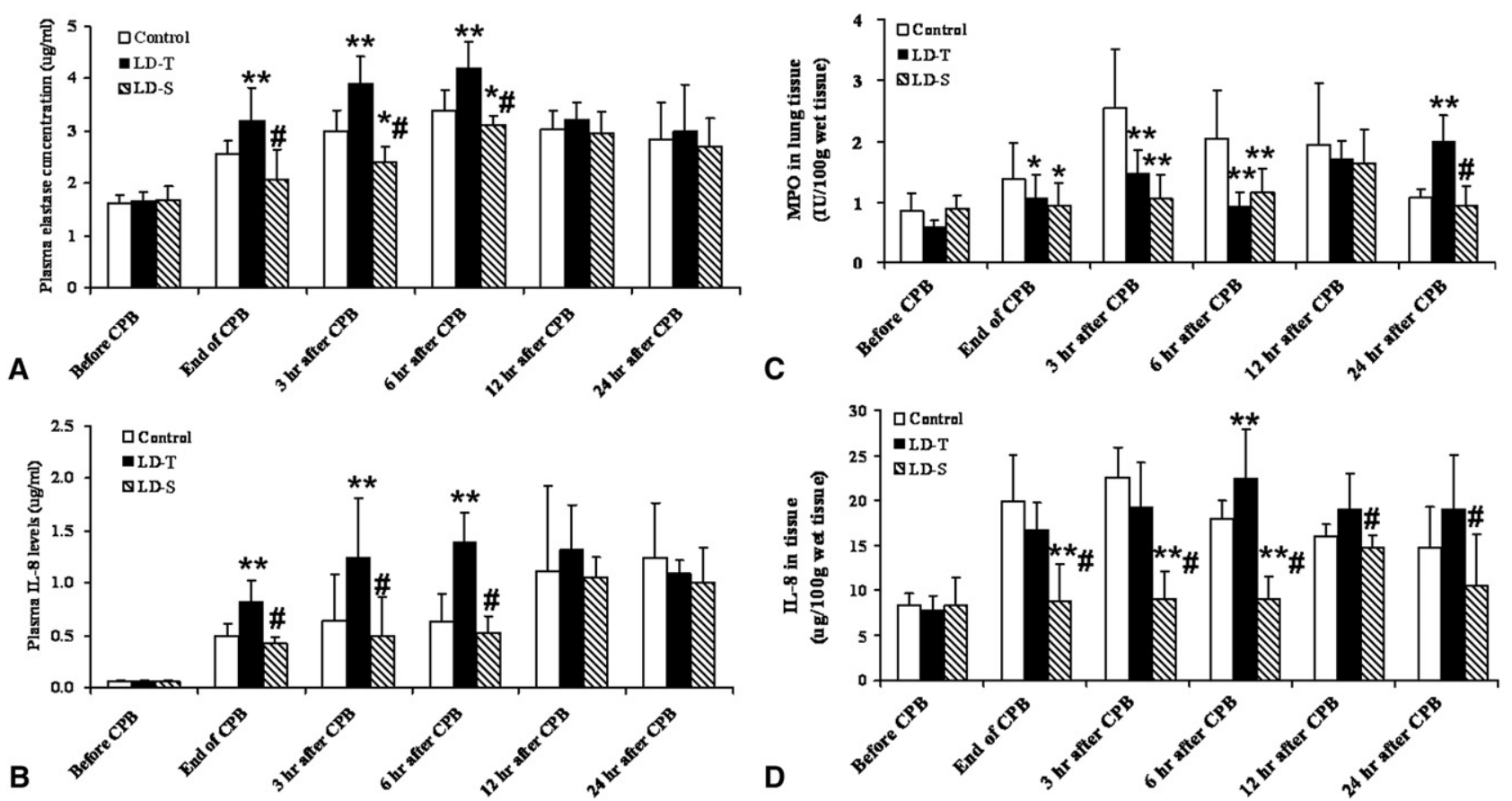

FIGURE 3. The plasma mediators and mediators in lung tissue of the 3 groups. A, The concentration of neutrophil elastase in the LD-T group was significantly higher than that in the control group at the end of and 3 and 6 hours after $\mathrm{CPB}(P<.01)$. The concentration of neutrophils elastase in the control group was significantly higher than that in the LD-S group at 3 hours $(P<.01)$ and 6 hours $(P<.01)$ after CPB. B, The plasma IL-8 levels in the LD-T group was significantly higher than those in the control group and LD-S group at the end of CPB $(P<.01)$ and 3 hours $(P<.01)$ and 6 hours $(P<.01)$ after CPB. No difference between the control and LD-S group was noticed. C, The concentrations of MPO in the 2 depleting groups were lower than that in the control group at the end of $\mathrm{CPB}(P=.041, P=.037)$ and 3 hours $(P<.01)$ and 6 hours after $\mathrm{CPB}(P<.01)$. The concentration of MPO in the LD-T group was significantly higher than that in the control group and LD-S group at 24 hours after CPB $(P<.01)$. D, The concentration of IL-8 in lung tissue in the LD-S group was significantly lower than that in the control group at the end of CPB $(P<.01)$ and 3 hours $(P<.01)$ and 6 hours after CPB $(P<.01)$. It was significantly lower than that in the LD-T group at the end of CPB and 3, 6, 12, and 24 hours after CPB $(P<.01)$. LD-T group, The leukocyte depletion filter was used from 3 minutes to the end of CPB; LD-S group, the leukocyte depletion filter was used from 3 minutes to 13 minutes of CPB; control group, no leukocyte depletion filter was used; $C P B$, cardiopulmonary bypass; $I L-8$, interleukin $8 ; M P O$, myeloperoxidase. $* P<.05 ; * * P<.01$ versus control group; $\# P<.01$ versus LD-T group.

filtration, and there was a reduction of $74.14 \%$ neutrophils when compared with the control group after 10 minutes of filtration was used. The figures were much higher than those reported by other researchers. ${ }^{7,15}$ We observed that the neutrophil count in the circulation was similar in the 2 leukocyte depletion groups $(P=.582)$ and remained so until the end of the study (24 hours after $\mathrm{CPB}$ ). These results suggested that short-term depletion was as technically feasible as the prolonged depletion strategy. We also observed that in our study the PVR in the short-term depletion group, a value that correlates with pulmonary vascular tone and pulmonary endothelial function after $\mathrm{CPB},{ }^{16}$ was significantly lower than that in the control and LD-T groups after CPB. The oxygen index in the LD-S group was also significantly higher than those in the control and LD-T groups at 24 hours after $\mathrm{CPB}$. All together, these findings suggested that the dogs in the LD-S group exhibited better pulmonary function than the dogs in the LD-T group. To explain these results, we hypothesized that leukocyte depletion strategy may play a crucial role for pulmonary protection.
Most studies in the literature on this subject used leukocyte depletion for the entire course of $\mathrm{CPB}$, with results that were not very satisfactory. ${ }^{3,5,8} \mathrm{We}$ speculated that the unsatisfactory results may be due to the following 3 reasons. First, the efficiency of leukocyte reduction reported by several other investigators was quite low, only $17.6 \%$ to $45 \%$. $^{7,15}$ Second, Ilmakunnas and colleagues ${ }^{17}$ found that neutrophil $\mathrm{CD} 11 \mathrm{~b}$ expression was actually increased by the leukocyte filter, with the implication that leukocyte filtration actually increased the activation of neutrophils, with ensuing magnification of the inflammatory response. Third, in vitro studies have shown that the highest leukocyte removal rate occurs during the first 15 minutes of $\mathrm{CPB},{ }^{18}$ followed by a gradual decline with time. In other words, after the first 15 minutes, the leukocyte depletion filter became less and less efficient in removing leukocytes, but became more and more of a focus of activation of the trapped neutrophils with magnification of the inflammatory process.

In this study, we assessed the levels of inflammatory mediators, namely NE and IL-8, in circulation. NE, which 

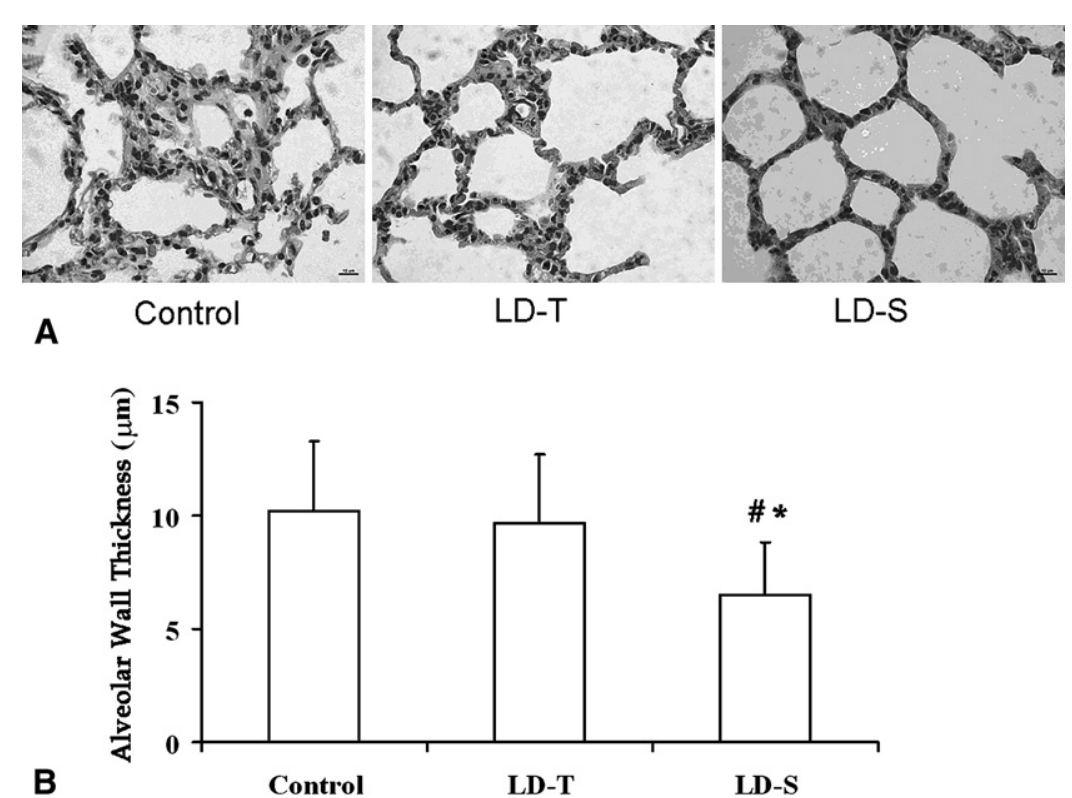

FIGURE 4. Pathologic examination of lung tissue. A, The pulmonary tissue in the 3 groups stained with hematoxylin and eosin examined under a light microscope at $\times 400$ magnification. B, The alveolar walls in the control group and LD-T group were significantly thicker than that in the LD-S group at 24 hours after CPB. LD-T group, The leukocyte depletion filter was used from 3 minutes to the end of CPB; LD-S group, the leukocyte depletion filter was used from 3 minutes to 13 minutes of $\mathrm{CPB}$; control group, no leukocyte depletion filter was inserted in the circuit; $C P B$, cardiopulmonary bypass. $* P<.001$ versus control group; $\# P<.001$ versus LD-T group.

was the marker of activation of neutrophils ${ }^{19}$ and was also one of several most important proteinases among various inflammatory mediators released during and after $\mathrm{CPB}$, was significantly higher in the LD-T group than in the LD-S and control groups. Even up until 6 hours after $\mathrm{CPB}$, the result was consistent with what Mihaljevic and associates ${ }^{20}$ had previously found. It suggested the possibility of a more extensive activation of neutrophils in the LD-T group caused by prolonged blood and filter contact, with continuous activation and release of inflammatory mediators by the trapped neutrophils during the entire course of CPB. Similarly, we also assessed the circulating levels of IL-8, a member of the CXC chemokine family known to function as an activator and chemoattractant to cause infiltration and degranulation of neutrophils ${ }^{21}$ and associated with numerous acute and chronic inflammatory disorders. ${ }^{22}$ The different IL-8 levels in the 3 groups again suggested that prolonged leukocyte filtration caused more severe inflammatory response than short-term filtration.

Pulmonary tissue is the target of the activated neutrophils and cytokines when it was reperfused; therefore, the levels of these activated neutrophils and cytokines in the lung tissue should be correlated directly with the degree of CPB-induced inflammatory response and, by further inference, the efficacy of various leukocyte depletion strategies on attenuation of ALI. In this study, the concentrations of MPO and IL-8 in the pulmonary tissue were measured during and after CPB. MPO is the marker of degranulation of neutrophils in plasma and also the marker of recruitment of neutrophils in tissue. ${ }^{23} \mathrm{~A}$ correlation between neutrophil degranulation and systemic organ failure after CPB has been reported. ${ }^{24}$ The results in our study showed that fewer neutrophils were sequestrated in the pulmonary tissue in the LD-S group than in the LD-T group. The thickness of alveolar wall, an indication of the degree of inflammatory response, was significantly thinner in the LD-S group than in the LD-T and control groups, again suggesting a lesser degree of inflammatory response in the LD-S group. It was interesting to note that while the circulating neutrophil counts in both the LD-S and LD-T groups during and at the end of CPB were more or less the same, the extent of inflammatory response in the plasma and lung tissue was vastly different. Thus circulating neutrophil count was not a reliable indicator for the degree of inflammatory response. It might explain the fact that with similar extents of leukocyte depletion, varying degrees of pulmonary protection have been observed by different investigators.

In conclusion, our study showed that both the short-term and prolonged leukocyte depletion strategies effectively reduced the circulating neutrophil count. However, shortterm leukocyte depletion appeared more effective in preserving pulmonary function. We speculate that the prolonged leukocyte-depletion strategy allows the filter to remain in the circuit with the trapped and activated neutrophils to continuously release injurious inflammatory mediators to the circulation and eventually to the reperfused lung tissue, causing much more inflammatory response and ALI. 


\section{References}

1. Ng CS, Wan S, Yim AP, Arifi AA. Pulmonary dysfunction after cardiac surgery. Chest. 2002;121:1269-77.

2. Ortolano GA, Capetandes A, Wenz B. A review of leukofiltration therapy for decreasing the morbidity associated with cardiopulmonary bypass and acute inflammatory bowel disease. Ther Apher. 2002;6:119-29.

3. Alexiou C, Tang AA, Sheppard SV, Smith DC, Gibbs R, Livesey SA, et al. The effect of leucodepletion on leucocyte activation, pulmonary inflammation and respiratory index in surgery for coronary revascularisation: a prospective randomised study. Eur J Cardiothorac Surg. 2004;26:294-300.

4. Olivencia-Yurvati AH, Ferrara CA, Tierney N, Wallace N, Mallet RT. Strategic leukocyte depletion reduces pulmonary microvascular pressure and improves pulmonary status postcardiopulmonary bypass. Perfusion. 2003;18:23-31.

5. Warren OJ, Tunnicliffe CR, Massey RM, Wallace S, Smith AJ, Alcock EM, et al. Systemic leukofiltration does not attenuate pulmonary injury after cardiopulmonary bypass. ASAIO J. 2008;54:78-88.

6. de Vries AJ, Gu YJ, Post WJ, Vos P, Stokroos I, Lip H, et al. Leucocyte depletion during cardiac surgery: a comparison of different filtration strategies. Perfusion. 2003; 18:31-8.

7. Baksaas ST, Videm V, Mollnes TE, Tølløfsrud S, Hetland G, Pedersen T, et al. Leucocyte filtration during cardiopulmonary bypass hardly changed leucocyte counts and did not influence myeloperoxidase, complement, cytokines or platelets. Perfusion. 1998;13:429-36.

8. Fabbri A, Manfredi J, Piccin C, Soffiati G, Carta MR, Gasparotto E, et al. Systemic leukocyte filtration during cardiopulmonary bypass. Perfusion. 2001; 16(suppl):11-8.

9. Gu YJ, de Vries AJ, Vos P, Boonstra PW, van Oeveren W. Leukocyte depletion during cardiac operation: a new approach through the venous bypass circuit. Ann Thorac Surg. 1999;67:604-9.

10. Matheis G, Scholz M, Simon A, Henrich D, Wimmer-Greinecker G, Moritz A. Timing of leukocyte filtration during cardiopulmonary bypass. Perfusion. 2001; 16(suppl):31-7.

11. Kaczka DW, Hager DN, Hawley ML, Simon BA. Quantifying mechanical heterogeneity in canine acute lung injury: impact of mean airway pressure. Anesthesiology. 2005;103:306-17.
12. Clark SC. Lung injury after cardiopulmonary bypass. Perfusion. 2006;21:225-8.

13. Messent M, Sullivan K, Keogh BF, Morgan CJ, Evans TW. Adult respiratory distress syndrome after cardiopulmonary bypass: incidence and prediction. Anaesthesia. 1992;47:267-8.

14. Tönz M, Mihaljevic T, von Segesser LK, Fehr J, Schmid ER, Turina MI. Acute lung injury during cardiopulmonary bypass. Are the neutrophils responsible? Chest. 1995;108:1551-6.

15. Nanson JK, Sheppard SV, Kulkarni M, Smith DC. A comparison of sequential total and activated white cell count in patients undergoing coronary artery bypass grafting, using cardiopulmonary bypass, with and without a white cell filter [abstract]. Crit Care. 1999;4(Suppl A):3.

16. Yun TJ, Rho JR. Aprotinin attenuates the elevation of pulmonary vascular resistance after cardiopulmonary bypass. J Korean Med Sci. 2006;21:25-9.

17. Ilmakunnas M, Pesonen EJ, Ahonen J, Ramo J, Siitonen S, Repo H. Activation of neutrophils and monocytes by a leukocyte-depleting filter used throughout cardiopulmonary bypass. J Thorac Cardiovasc Surg. 2005;129: 851-9.

18. Gourlay T, Fleming J, Taylor KM. Laboratory evaluation of the Pall LG6 leucocyte depleting arterial line filter. Perfusion. 1992;7:131-40.

19. Gu YJ, Schoen P, Tigchelaar I, Loef BG, Ebels T, Rankin AJ, et al. Increased neutrophil priming and sensitization before commencing cardiopulmonary bypass in cardiac surgical patients. Ann Thorac Surg. 2002;74: 1173-9.

20. Mihaljevic T, Tönz M, von Segesser LK, Pasic M, Grob P, Fehr J, et al. The influence of leukocyte filtration during cardiopulmonary bypass on postoperative lung function. J Thorac Cardiovasc Surg. 1995;109:1138-45.

21. Singer M, Sansonetti PJ. IL-8 is a key chemokine regulating neutrophi recruitment in a new mouse model of Shigella-induced colitis. J Immunol. 2004;173:4197-206.

22. Baggiolini M. Chemokines and leukocyte traffic. Nature. 1998;392:565-8.

23. Bradley PP, Priebat DA, Christensen RD, Rothstein G. Measurement of cutaneous inflammation: estimation of neutrophil content with an enzyme marker. $J$ Invest Dermatol. 1982;78:206-9.

24. Boyle EM, Pohlman TH, Johnson MC, Verrier ED. The systemic inflammatory response. Ann Thorac Surg. 1997;64:31-7. 